\title{
¿Existe Alteración de los Mecanismos de Degradación del Colágeno de la Matriz Extracelular Manifestada en la Expresión de MMP2 y TIMP2 en Pacientes con Hernia Inguinal Primaria?
}

\author{
Does Alteration of Extracellular Matrix Collagen Degradation Mechanisms Manifested in the \\ Expression of MMP2 and TIMP2 in Patients with Primary Inguinal Hernia Exists? \\ "Víctor Molina; "Carlos Manterola \& ** Grupo MINCIR
}

MOLINA, V.; MANTEROLA, C. \& GRUPO MINCIR. ¿Existe alteración de los mecanismos de degradación del colágeno de la matriz extracelular manifestada en la expresión de MMP2 y TIMP2 en pacientes con hernia inguinal primaria? Int. J. Morphol., 30(2):683687, 2012.

RESUMEN: Existe evidencia de asociación de alteraciones de la matriz colágena en sujetos con hernia inguinal (HI). El objetivo de este estudio fue determinar la existencia de alteraciones en la expresión de MMP2 y TIMP2 en sujetos con HI primaria. Estudio de casos y controles, realizado en los Servicios de Cirugía y de Urgencias del Hospital Regional de Temuco entre 2009-2010. Se incluyeron pacientes con HI primaria mayores de 15 años, sin restricción de género (casos), e Individuos sin hernias, mayores de 15 años, intervenidos por apendicitis aguda (controles). Se excluyeron portadores de condiciones predisponentes al desarrollo de hernias de pared abdominal. La variable resultado fue determinación de MMP2 y TIMP2 mediante inmunohitoquímica (IHQ). Otras variables de interés estudiadas fueron: hábito tabáquico, antecedente familiar de hernia, historia de estreñimiento, peso, estatura e índice de masa corporal (IMC). Se utilizó estadística descriptiva; analítica y modelos de regresión logística. Se estudiaron 21 casos y 20 controles. El $73 \%$

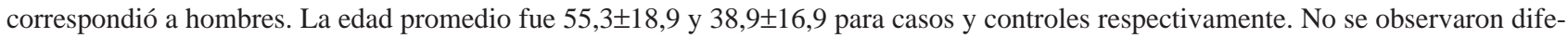
rencias estadísticamente significativas en las variables sexo, estatura, peso, IMC y hábito tabáquico entre casos y controles. Se verificaron diferencias significativas en las variables, antecedente familiar de hernia e historia de estreñimiento (más frecuentes en casos). La tinción IHQ de MMP2, fue mayor en los casos (53,4\% vs. 40,0\%; p=0,5068), y la de TIMP2, fue mayor en los controles (35,0\% vs. 23,8\%; $\mathrm{p}=0,7333)$. El análisis multivariado descartó confundentes. Con la metodología utilizada no se verificó asociación entre alteración de los mecanismos de degradación del colágeno, manifestados en la expresión de MMP2 y TIMP2, con el desarrollo de HI primaria.

PALABRAS CLAVE: Hernia; Hernia inguinal; Matriz colágena; Colágeno; Metaloproteinasas; Inmunohitoquímica; Estudio de casos y controles.

\section{INTRODUCCIÓN}

La hernia inguinal (HI), representa el $80 \%$ de las hernias pared abdominal y constituye una de las principales causas de consulta quirúrgica tanto electiva como de urgencia (Pérez et al., 1999).

Respecto a la patogenia de la HI, existen diversos reportes que la asocian a alteraciones a nivel de tejido conjuntivo y de la matriz colágena (Wagh et al., 1972; Friedman et al., 1993; Rosch et al., 2002; Bórquez et al., 2003; Bendavid, 2004). Posteriormente, han aparecido estudios que han establecido mayor expresión de tipos específicos de metaloproteinasas, enzimas que degradan colágeno, en cultivos de fibroblastos de individuos portadores de $\mathrm{HI}$
(Bellón et al., 1997; Bellón et al., 2001); aunque se trata de artículos de tipo experimental sin una estimación del tamaño de la muestra y calidad metodológica inadecuada.

Se ha observado también menor síntesis de colágeno medida a través de la incorporación de prolina radiomarcada en cultivos de fibroblastos en sujetos con HI que en controles (Bellón et al., 2001); variaciones en el diámetro y periodicidad de las fibras colágenas de la vaina de los rectos, fascia transversalis, piel y peritoneo en individuos con $\mathrm{HI}$ (Broggi, 1982; Pans et al., 2001; Rodrigues Junior et al., 2002; Ozdogan et al., 2006); así como también en portadores de aneurisma de aorta abdominal y hernias abdominales

\footnotetext{
* Programa de Magíster y Doctorado en Ciencias Médicas, Facultad de Medicina, Universidad de La Frontera, Temuco, Chile.

** Departamento de Cirugía y Traumatología, Facultad de Medicina, Universidad de La Frontera, Temuco, Chile.
} 
(Antoniou et al., 2011). Finalmente, estudios realizados en nuestro medio lograron objetivar alteraciones en la relación de los colágenos tipo I y III medidos por tinciones específicas y por inmunohistoquímica (IHQ) (Bórquez et al.; Bórquez, 2005).

El catabolismo del colágeno se realiza por enzimas proteolíticas producidas por fibroblastos; éstas, se denominan en conjunto metaloproteinasas de la matriz (MMPs) (Antoniou et al.; Aren et al., 2001). En estudios realizados en cultivo de fibroblastos de fascia transversalis de pacientes portadores de HI primaria se identificó un incremento de la expresión de MMP2, en comparación con sujetos sin HI (Bellón et al., 1997; Bellón et al., 2001).

Por otra parte, existen inhibidores titulares para las MMP. El inhibidor específico para la MMP2 es el TIMP-2. Se ha reportado menor expresión de esa molécula en fascia transversalis de individuos con HI directa (Abci et al., 2005). $\mathrm{Si}$ bien, este estudio carece de rigurosidad metodológica, sus resultados permiten establecer una razonable duda acerca del rol que podrían jugar los mecanismos de degradación del colágeno en la génesis de la HI.

El objetivo de este estudio es determinar la existencia de alteraciones en la expresión de MMP2 y su inhibidor tisular (TIMP2) en piel sujetos con HI primaria.

\section{MATERIAL Y MÉTODO}

Diseño del estudio: Estudio de casos y controles.

Centro: El estudio fue realizado en los Servicios de Cirugía y de Urgencias del Hospital Hernán Henríquez Aravena de Temuco, en el período 2009-2010.

Participantes: Sujetos con HI primaria mayores de 15 años, sin restricción de género; los que en adelante se denominan como "casos". Individuos sin hernias la pared abdominal intervenidos por apendicitis aguda, mayores de 15 años, sin restricción de género; los que en adelante se denominan como "controles". Se realizó emparejamiento por sexo entre casos y controles. Se excluyeron sujetos portadores de condiciones que predisponen al desarrollo de hernias de pared abdominal como obesidad, desnutrición, enfermedades genéticas del tejido conjuntivo y neoplasias.

Tratamiento: Los casos fueron tratados de su HI mediante intervención quirúrgica, realizándose en todos ellos hernioplastia con malla de polipropileno, con técnica de Lichtenstein.
Protocolo de estudio: Se tomó una muestra de piel de 10 por $3 \mathrm{~mm}$. desde el sitio de la incisión que abarcó en profundidad hasta la hipodermis (tanto en los casos como en los controles). Las muestras se conservaron en formalina tamponada hasta ser procesadas en el Servicio de Anatomía Patológica del Hospital Hernán Henríquez Aravena. Allí, fueron sometidas a pruebas de inmunohistoquímica para determinar los niveles de MMP2 y TIMP2.

Variables: La variable resultado o principal fue "determinación de MMP2 y TIMP2 mediante IHQ". Dado que no existe un instrumento validado que permita objetivar los resultados en este tipo de pruebas, sus resultados son dependientes del entrenamiento del observador. Para este efecto se contó con la participación de un médico patólogo con vasta experiencia en mediciones IHQ. Los resultados fueron clasificados con asignación de 1 a 3 cruces dependiendo de la intensidad de tinción. Se le asignó 1 cruz a las muestras que no presentaron tinción interpretándose como ausencia de la molécula buscada. Se asignaron 3 cruces a la máxima tinción correspondiendo a la mayor expresión de la molécula. Un rango intermedio lo constituyeron las muestra que aunque dieron tinción positiva indicando presencia de la molécula, la intensidad con que lo hicieron fue menor.

Otras variables de interés estudiadas: hábito tabáquico, antecedente familiar de hernia, historia de estreñimiento, peso, estatura e índice de masa corporal (IMC).

Tamaño de la muestra: Basado en la estimación de la diferencia de media para los valores de MMP2 medidos en pacientes con y $\sin \mathrm{HI}$, con un nivel de significancia de $5 \%$ y un poder de $80 \%$ se estimó una muestra de 19 individuos por grupo. En relación a la medición de TIMP2 se calculó, en base a la diferencia de porcentajes en sujetos con y sin HI, con un nivel de significancia de $5 \%$ y un poder de $80 \%$, la necesidad de una muestra de 20 pacientes por grupo. Por ser este último el tamaño de muestra mayor, nos quedamos con él para el desarrollo de esta investigación.

Métodos estadísticos: Los datos fueron analizados con un programa Stata 10.0/SE ®. Después de realizar un análisis exploratorio de los datos, se aplicó estadística descriptiva con cálculo de porcentajes, medidas de tendencia central y dispersión. Posteriormente, se realizaron análisis inferenciales, examinando asociación entre las variables, mediante prueba de chi cuadrado de Pearson para variables categóricas y T-test para variables continuas. Finalmente, se realizaron cálculos de magnitud de la asociación.

Aspectos éticos: El protocolo de estudio fue aprobado por el Comité de Ética del Servicio de Salud Araucanía Sur, Temuco, Chile. 


\section{RESULTADOS}

La muestra estuvo compuesta por 21 casos y 20 controles. El $73 \%$ correspondió a hombres. La edad promedio fue mayor en los casos que en los controles $(55,3 \pm 18,9$ y $38,9 \pm 16,9$ respectivamente). No se observaron diferencias estadísticamente significativas en las variables sexo, estatura, peso, IMC y hábito tabáquico entre casos y controles. Se verificaron diferencias significativas en las variables, antecedente familiar de hernia $(0,0272)$ e historia de estreñimiento $(0,0491)$; más frecuentes en casos que controles (Tabla I).
La tinción IHQ de MMP2, fue mayor en los casos (53,4\% vs. $40,0 \%)$, diferencia no estadísticamente significativa ( $\mathrm{p}=0,5068)$. Por su parte, la tinción IHQ de TIMP2, fue mayor en los controles ( $35,0 \%$ vs. $23,8 \%$ ), diferencia no estadísticamente significativa ( $\mathrm{p}=0,7333)$. Tabla II.

En el análisis estratificado aparecen como posibles confundentes las variables sexo, antecedente de tabaquismo, historia de estreñimiento y antecedente familiar de hernia. Se realizó un análisis controlando por cada una de estas variables sin encontrar cambios en los resultados, por lo que, se descarta un efecto confundente de estas variables en los resultados.

Tabla I. Distribución de las variables en estudio entre casos y controles.

\begin{tabular}{|c|c|c|c|c|}
\hline \multicolumn{2}{|l|}{ Variable } & Casos $(n=21)$ & Controles $(\mathbf{n}=\mathbf{2 0})$ & $\mathbf{p}$ \\
\hline \multicolumn{2}{|l|}{ Edad (años) } & $55,3 \pm 18,9$ & $38,9 \pm 16,9$ & 0,0122 \\
\hline \multicolumn{2}{|l|}{ Estatura (cm) } & $163,8 \pm 6,9$ & $165,0 \pm 7,6$ & 0,6721 \\
\hline \multicolumn{2}{|l|}{ Peso (Kg) } & $71,3 \pm 11,5$ & $72,6 \pm 11,7$ & 0,5968 \\
\hline \multicolumn{2}{|l|}{ IMC } & $26,6 \pm 3,9$ & $26,4 \pm 3,0$ & 0,9879 \\
\hline \multirow[t]{2}{*}{$\operatorname{Sexo}(\%)$} & Femenino & 19,1 & 35,0 & \multirow[b]{2}{*}{0,2123} \\
\hline & Masculino & 80,9 & 65,0 & \\
\hline \multicolumn{2}{|c|}{ Hábito tabáquico (\%) } & 80,9 & 65,0 & 0,1767 \\
\hline \multicolumn{2}{|c|}{ Antecedente familiar de hernia $(\%)$} & 23,8 & 0,0 & 0,0272 \\
\hline \multicolumn{2}{|c|}{ Historia de estreñimiento (\%) } & 19,4 & 0,0 & 0,0491 \\
\hline
\end{tabular}

Tabla II. Distribución de las tinciones de IHQ entre casos y controles.

\begin{tabular}{lcccc}
\hline Variable & & Casos $(\mathbf{n}=\mathbf{2 1})$ & Controles $(\mathbf{n = 2 0})$ & p \\
\hline Tinción IHQ MMP2 (\%) & $(+++)$ & 53,4 & 40,0 & \\
& $(++)$ & 19,0 & 35,0 & 0,5068 \\
& $(+)$ & 28,6 & 25,0 & \\
Tinción IHQ TIMP2 (\%) & $(+++)$ & 23,8 & 35,0 & \\
& $(++)$ & 23,8 & 20,0 & 0,7333 \\
& $(+)$ & 52,4 & 45,0 & \\
\hline
\end{tabular}

\section{DISCUSIÓN}

La evidencia disponible sugiere que la etiología y el tratamiento de las hernias se basan en la comprensión de un defecto mecánico que requiere una reparación confiable. La necesidad de conocimiento adicional relacionado con los fenómenos fisiopatológicos y patogénicos, no constituye un objetivo generalizado en la comunidad quirúrgica, porque los cirujanos carecen de tradición en investigación (al menos aquella aplicada al laboratorio).
Afortunadamente, anatomistas, biólogos moleculares, bioquímicos, patólogos, y otros, han estudiado la matriz colágena y enzimas; la asociación con factores ambientales y hereditarios como el hábito tabáquico, enfermedades congénitas y anomalías cromosómicas; y la asociación entre estos y el desarrollo de hernias de la pared abdominal.

A propósito de ello, se ha demostrado en diferentes 
estudios la existencia de alteraciones en la composición y distribución de las fibras colágenas en fascia transversalis y en piel de individuos portadores de hernias de pared abdominal (Abci et al.; Bórquez et al.; Ozdogan et al.; Pans et al.; Rodrigues Junior et al.)

Estudios más recientes han descrito la existencia de alteraciones en la expresión de enzimas involucradas en la remodelación del tejido conjuntivo, postulándose como el origen de los cambios en la composición y distribución del colágeno en portadores de hernia de pared abdominal (Antoniou et al., 2009; Aren et al.; Bellón et al., 1997; Bellón et al., 2001; Klinge et al., 1999); siendo la literatura existente al respecto escasa.

Los resultados de este estudio no son concordantes con aquellas publicaciones. No fue posible demostrar diferencias en la expresión de MMP2 ni TIMP2 en piel de individuos portadores y no portadores de HI primaria. Sólo se encontraron diferencias en variables que clásicamente han sido relacionadas con el desarrollo de HI. Sin embargo, al controlar por estas variables las diferencias en la expresión de MMP2 y TIMP2 entre casos y controles no presentó modificación significativa.

Por otra parte, en un estudio de casos y controles publicado recientemente; en el que se midieron concentraciones de MMP2 y TIMP2 en sujetos con HI, aneurisma de aorta abdominal y controles; se verificó que las concentraciones de MMP2 fueron inferiores en plasma de pacientes con HI y aneurisma aórtico que en los controles; y que los niveles de TIMP2 fueron significativamente ma- yores en individuos con HI que en los sujetos con aneurisma aórtico y los controles (Antoniou et al., 2011).

Es posible que los estudios hechos en laboratorio no logren emular la complejidad de las interacciones moleculares que ocurren in vivo. Por otra parte, los pequeños tamaños de muestra en dichos estudios nos obligan a mirar con ojos más críticos sus resultados, que probablemente en poblaciones o muestras mayores no se presenten.

Es necesario, al mismo tiempo, reconocer las limitaciones del diseño de casos y controles; que dicen relación fundamentalmente con la definición de casos y controles; y la alta probabilidad de caer en sesgos de distinto tipo, que a su vez pueden determinar confusión en la asociación en estudio (Manterola, 2001; Manterola, 2009). El tamaño de la muestra obtenido a partir de los datos existentes en los estudios previos puede ser insuficiente para estudios en que las diferencias reales entre dos poblaciones sean pequeñas. La interacción de múltiples factores en la génesis de las hernias de pared abdominal quizá justifique una selección y emparejamiento más exhaustivo de los grupos, con tamaños de muestra mayores, con el fin de evitar la interacción de variables confundentes.

Considerando lo anterior, y valorando en su justo mérito el diseño, podemos decir que a la luz de los resultados no es posible plantear una relación entre las alteraciones en los mecanismos de degradación del colágeno, manifestados en la expresión de MMP2 y TIMP2, con el desarrollo de HI primarias.

MOLINA, V.; MANTEROLA, C. \& GRUPO MINCIR. Does alteration of extracellular matrix collagen degradation mechanisms manifested in the expression of MMP2 and TIMP2 in patients with primary inguinal hernia exists? Int. J. Morphol., 30(2):683,687, 2012.

SUMMARY: There is some evidence of association b collagen matrix alterations in patients with inguinal hernia (IH). The aim of this study was to determine the existence of alterations in the expression of MMP2 and TIMP2 in patients with primary IH. Casecontrol study conducted in the Department of Surgery and Emergency of Temuco Regional Hospital in 2009-2010 periods. We included subjects with primary IH over 15 years, without gender restriction (cases) and subjects without hernias, aged 15, underwent surgery for acute appendicitis (controls). Patients with conditions predisposing to the development of abdominal wall hernias were excluded. End point was determination of MMP2 and TIMP2 by Immunohistochemistry (IHC). Other studied variables were: smoking, family history of hernia, history of constipation, weight, height and body mass index (BMI). Descriptive statistics, analytical and logistic regression models were used. 21 cases and 20 controls were studied. $73 \%$ were men. The average age was $55.3 \pm 18.9$ and $38.9 \pm 16.9$ for cases and controls respectively. There were no statistically significant differences in the variables sex, height, weight, BMI and smoking between cases and controls. Significant difference was found in the variables, family history of hernia and history of constipation (more common in cases). IHC staining of MMP2 was higher in cases $(53.4 \%$ vs. $40.0 \%, \mathrm{p}=0.5068)$ and IHC of TIMP2 was higher in controls (35.0\% vs. $23.8 \%, \mathrm{p}=0.7333)$. Multivariate analysis discarded confounders. With the methodology used has not been tested association between alteration of mechanisms of collagen degradation, manifested in MMP2 and TIMP2 expression with primary IH development.

KEY WORDS: Hernia; Inguinal hernia, Collagen Type I; Tissue Inhibitor of Metalloproteinases; Immunohistochemistry; Case-Control Studies. 


\section{REFERENCIAS BIBLIOGRÁFICAS}

Abci, I.; Bilgi, S. \& Altan, A. Role of TIMP2 in fascia transversalis on development of inguinal hernias. J. Invest. Surg., 18(3):123$8,2005$.

Antoniou, S. A.; Antoniou, G. A.; Granderath, F. A. \& Simopoulos, C. The role of matrix metalloproteinases in the pathogenesis of abdominal wall hernias. Eur. J. Clin. Invest., 39(11):953-9, 2009.

Antoniou, G. A.; Tentes, I. K.; Antoniou, S. A.; Georgiadis, G. S.; Giannoukas, A. D.; Simopoulos, C. \& Lazarides, M. K. Circulating matrix metalloproteinases and their inhibitors in inguinal hernia and abdominal aortic aneurysm. Int. Angiol., 30(2):123-9, 2011.

Aren, A.; Gökçe, A. H.; Gökçe, F. S. \& Dursun, N. Roles of matrix metalloproteinases in the etiology of inguinal hernia. Hernia, 15(6):667-71, 2011.

Bellón, J. M.; Buján, J.; Honduvilla, N. G.; Jurado, F.; Gimeno, M. J.; Turnay, J.; Olmo, N. \& Lizarbe, M. A. Study of biochemical substrate and role of metalloproteinases in fascia transversalis from hernia processes. Eur. J. Clin. Invest., 27(6):510-6, 1997.

Bellón, J. M.; Bajo, A.; Ga-Honduvilla, N.; Gimeno, M. J.; Pascual, G.; Guerrero, A. \& Buján, J. Fibroblasts from the transversalis fascia of young patients with direct inguinal hernias show constitutive MMP2 over expression. Ann. Surg., 233(2):28791, 2001

Bendavid, R. The unified theory of hernia formation. Hernia, 8(3):171-6, 2004.

Bórquez, P., Garrido, L.; Manterola, C.; Peña, P.; Schlageter, C.; Orellana, J. J.; Ulloa, H. \& Peña, J. L. Study of collagen and elastic fibers of connective tissue in patients with and without primary inguinal hernia. Rev. Med. Chil., 131(11):1273-9. 2003.

Bórquez, P. Immunohistochemical measurement of type I and III collagen content of the skin of patients with primary inguinal hernia. Rev. Chil. Cir., 57:489-94. 2005.

Broggi, M. A. Hernia inguinocrural. Barcelona, JIMS, 1982.

Friedman, D. W.; Boyd, C. D.; Norton, P.; Greco, R. S.; Boyarsky, A. H.; Mackenzie, J. W. \& Deak, S. B. Increases in type III collagen gene expression and protein synthesis in patients with inguinal hernias. Ann. Surg., 218(6):754-60, 1993.

Klinge, U.; Zheng, H.; Si, Z.; Schumpelick, V.; Bhardwaj, R. S.; Muys, L. \& Klosterhalfen, B. Expression of the extracellular matrix proteins collagen I, collagen III and fibronectin and matrix metalloproteinase- 1 and -13 in the skin of patients with inguinal hernia. Eur. Surg. Res., 31(6):480-90, 1999.
Manterola, C. Estrategias de investigación. Diseños observacionales 2a parte. Estudio de casos y controles. Rev. Chil. Cir., 53:31620,2001

Manterola, C. Estudios observacionales. Los diseños utilizados con mayor frecuencia en investigación clínica. Rev. Med. Clin. Condes, 20:539-48, 2009.

Ozdogan, M.; Yildiz, F.; Gurer, A.; Orhun, S.; Kulacoglu, H. \& Aydin, R. Changes in collagen and elastic fiber contents of the skin, rectus sheath, transversalis fascia and peritoneum in primary inguinal hernia patients. Bratisl. Lek. Listy, 107(67):235-8, 2006.

Pans, A.; Albert, A.; Lapière, C. M. \& Nusgens, B. Biochemical study of collagen in adult groin hernias. J. Surg. Res., 95(2):107-13, 2001.

Pérez, P.; Amat, J. \& Korn, O. Hernias de la región inguinal. En hernias abdominales. Santiago, Publicaciones Mediterráneo Ltda., 1999. pp.47-77.

Rodrigues Junior, A. J.; Rodrigues, C. J.; da Cunha, A. C. \& Jin, Y. Quantitative analysis of collagen and elastic fibers in the transversalis fascia in direct and indirect inguinal hernia. Rev. Hosp. Clin. Fac. Med. Sao Paulo, 57(6):265-70, 2002.

Rosch, R.; Klinge, U.; Si, Z.; Junge, K.; Klosterhalfen, B. \& Schumpelick, V. A role for the collagen I/III and MMP-1/-13 genes in primary inguinal hernia? BMC Med. Genet., 3:2, 2002.

Wagh, P. V.; Leverich, A. P.; Sun, C. N.; White, H. J. \& Read, R. C. Direct inguinal herniation in men: a disease of collagen. $J$. Surg. Res., 17(6):425-33, 1974.

\section{Dirección para correspondencia: \\ Dr. Carlos Manterola \\ Departamento de Cirugía \\ Universidad de La Frontera \\ Temuco \\ CHILE}

Teléfono: $56-45-325760$

Fax: $\quad$ 56-45-325761

E-mail: cmantero@ufro.cl

Recibido : 09-04-2012

Aceptado: 08-05-2012 